\title{
Tranexamic for Treatment of Melasma
}

\section{Khaled Gharib* and Mohamed Nasr}

Department of Dermatology and Venereology, Faculty of Medicine, Zagazig University, Egypt

Melasma is a relatively common, acquired facial skin disorder of hyperpigmentation. It occurs in both sexes; although nearly $90 \%$ of the patients are female [1]. It is characterized by tan-brown macules and patches with a predilection for sun exposed areas, in particular the cheeks, forehead, upper lip, nose, and chin. Women are more affected than men (female to male ratio, 9:1) [2].

Melasma is encountered in all skin types, but is particularly seen in women with Fitzpatrick skin types IV to VI. The pathogenesis of melasma is not fully understood. Genetic background and sun exposure seems to be the most important etiologic factors besides pregnancy, systemic drugs, hormonal medications, phototoxic or photoallergic cosmetics and autoimmune thyroid disease. Sunlight exposure appears to be essential for its development. Melasma is often difficult to manage because of its refractory and recurrent nature [3].

Hydroquinone has been considered as the gold standard for treatment. Other treatments include mequinol, tretinoin, kojic acid, glycolic acid, ascorbic acid, or various combinations of these medications. Although a variety of therapeutic options are currently available, no treatment has been proved to be consistently satisfactory [1].

Tranexamic acid (trans-4-aminomethyl cyclohexanecarboxylic acid: TNA) is a synthetic lysine analog with antifibrinolytic effect through the reversible blockade of lysine-binding sites on plasminogen molecules. Due to its activity as a plasmin inhibitor, oral TNA has been originally utilized in reducing blood loss for management of menorrhagia or major surgery. In the dermatological field, it has been used as a topical agent or intradermal injection in melasma patients for its whitening effects (Figure 1) [4].

It is known that UV irradiation induces plasmin activator synthesis and plasmin activity in cultured keratinocyte. Plasmin activated precursors of secretory phospholipase A2, which participates in the production of arachidonic acid from membrane phospholipids. Arachidonic acid is a precursor to prostaglandins E2 and leukotrienes, which can subsequently lead to melanogenesis (Figure 2) $[5,6]$.

The total duration of the treatment is 12 weeks. At baseline, a photo is taken and a topical anesthetic is applied on both sides of the face for 20 to 30 minutes under occlusion before the injection. A 30-gauge, $0.5 \mathrm{~mL}$ insulin syringe with $4 \mathrm{mg} / \mathrm{mL}$ TNA $(\mathrm{pH} 6.5-8.0)$ is prepared under sterile conditions. Injections of about $0.05 \mathrm{~mL}$ were applied intradermally on the melasma lesion at $1 \mathrm{~cm}$ intervals. This is repeated weekly for 12 weeks. The patients are advised to avoid excessive sun exposure and to apply a broad spectrum sunscreen with a sun protection factor of 15 or higher in the morning [1].

A detailed questionnaire is prepared to record the subjects' histories, sunlight exposures, related medicine administrations, former treatments, cosmetics usage, related habits, and the like that will be helpful in determining the causes of melasma. An explanation including the risks, benefits, and potential complications are given to the patients, and written informed consent is obtained from each. The size and severity of each melasma will be recorded by Melasma area and severity index (MASI). Patients are instructed to return to the outpatient clinic at weeks $4,8,12$ for analysis of the changes in the melasma and in each visit photo will taken. We will also instruct the patients to apply broad-spectrum sunscreens, to avoid over scrubbing of the face in daily skin care, and to stop any other therapies during the treatment period [6].

Melasma Area and Severity Index (MASI) is a scoring system used to quantify the severity of melasma. Four areas of the face are evaluated: forehead (F), right malar region (MR), left malar region (ML) and chin (C), corresponding to $30 \%, 30 \%, 30 \%$, and $10 \%$ of the total face respectively. Amount of pigmentation involved by melasma in these four areas (AF, AMR, AML, and AC) is graded as a numerical value: 0 : no involvement; 1 : less than $10 \%$ involvement; 2 : $10-29 \%$; 3 : 30-49\%; 4: 50-69\%; 5: 70-89\%; and 6: 90-100\%. Severity of melasma is graded upon two factors; darkness (D) of melasma compared to the normal skin and homogeneity $(\mathrm{H})$ of hyperpigmentation [1].

These are assessed on a scale from 0 to 4 . The rating scale for both darkness and homogeneity of melasma is as follows; 0: absent; 1 : slight;

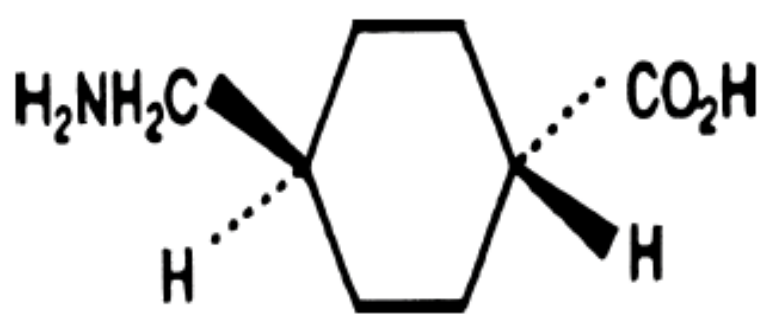

Figure 1: Chemical structure of Tranexamic acid.

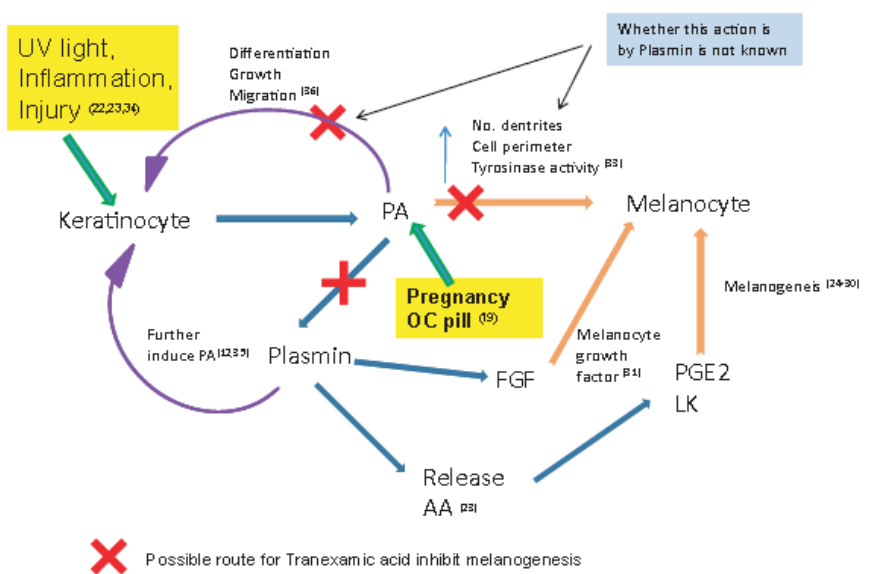

Figure 2: Mechanism of action of TNA in melanogenesis.

*Corresponding author: Khaled Gharib, Zagazig University, Zagazig, Egypt, Tel: 00201001658084; E-mail: kh_gharib@hotmail.com

Received March 20, 2015; Accepted May 19, 2015; Published May 28, 2015

Citation: Gharib K, Nasr M (2015) Tranexamic for Treatment of Melasma. Pigmentary Disorders 2: 190. doi:10.4172/2376-0427.1000190

Copyright: ( 2015 Gharib K, et al. This is an open-access article distributed under the terms of the Creative Commons Attribution License, which permits unrestricted use, distribution, and reproduction in any medium, provided the original author and source are credited. 
2: mild; 3: marked; and 4: maximum. MASI score is then calculated according to the following formula:

$\mathrm{MASI}=0.3(\mathrm{DF}+\mathrm{HF}) \mathrm{AF}+0.3(\mathrm{DMR}+\mathrm{HMR}) \mathrm{AMR}+0.3(\mathrm{DML}$ $+\mathrm{HML}) \mathrm{AML}+0.1(\mathrm{DC}+\mathrm{HC}) \mathrm{AC}$.

The maximum score for MASI can be 48 while minimum can be 0 . Patient satisfaction score is subjectively graded on the basis of four point scale: excellent, good, fair, and poor. Possible side effects of TNA are slight erythema and edema [4].

\section{References}

1. Sun YLQ, Fu ZH, He C, He Z, Fu L, et al. (2014) Treatment of melasma with oral administration of compound tranexamic acid: a preliminary clinical trial. JEADV 28: 388-394.
2. Guevara L, Pandya G (2003) Safety and efficacy of $4 \%$ hydroquinone combined with $10 \%$ glycolic acid, antioxidants, and sunscreen in the treatment of melasma. Int J Dermatol 42: 966-972.

3. Gupta K, Gover D, Nouri K, Taylor S (2006) The treatment of melasma: a review of clinical trials. J Am Acad Dermatol 55: 1048-1065.

4. $\mathrm{CHO} \mathrm{HH}, \mathrm{CHOI} M, \mathrm{CHO}$ S, Lee JH (2012) Role of oral tranexamic acid in melasma patients treated with IPL and low fluence QS Nd:YAG laser. J Dermatol Treat $1-5$.

5. Budamakuntla L, Loganathan E, Suresh H, Shanmugam S, Suryanarayan $S$, et al (2013) A randomised, open-label, comparative study of tranexamic acid microinjections and tranexamic acid with microneedling in patients with melasma. J Cutan Aesthet Surg. 6: 139-143.

6. Tse W, Hui E (2013) Tranexamic acid: an important adjuvant in the treatment of melasma. J Cosmet Dermatol 1: 57-66. 